\title{
Post-acne Erythema Successfully Treated with 595-nm Picosecond-domain Neodymium:Yttrium-aluminum-garnet Laser
}

Jae-Hong Kim ${ }^{1}$
Sung Jay Choe
Tae-Gyun Kim²

${ }^{1}$ Yonsei Joeun Dermatology Clinic, Seoul, Korea ${ }^{2}$ Department of Dermatology, Severance Hospital, Cutaneous Biology Research Institute, Yonsei University College of Medicine, Seoul, Korea
Received February 10, 2020

Accepted February 26, 2020

\section{Correspondence}

Tae-Gyun Kim

Department of Dermatology, Severance Hospital, Cutaneous Biology Research Institute, Yonsei University College of Medicine, 50-1 Yonsei-ro, Seodaemun-gu, Seoul 03722, Korea

Tel.: +82-2-2228-2080

Fax: +82-2-393-9157

E-mail: tgmed83ayuhs.ac

(C) Korean Society for Laser Medicine and Surgery

(@) This is an open access article distributed under the terms of the Creative Commons Attribution NonCommercial License (http://creativecommons.org/ licenses/by-nc/4.0) which permits unrestricted noncommercial use, distribution, and reproduction in any medium, provided the original work is properly cited.
Post-acne erythema (PAE) is one of the major problems during or after treatment of acne vulgaris with any modality. A variety of therapeutic options have been described with various clinical outcomes and side effects. We report here on treating a patient with PAE using $595-\mathrm{nm}$ picosecond-domain neodymium:yttrium-aluminum-garnet ( $\mathrm{Nd}: Y A G)$ laser. After four sessions of treatment, the patient displayed nearly complete improvement and no remarkable adverse effects. To the best of the authors' knowledge, this is the first case of the effective treatment of PAE using a 595- $\mathrm{nm}$ picosecond-domain Nd:YAG laser. We recommend that using a 595-nm picosecond-domain $\mathrm{Nd}$ :YAG laser can be both an effective and safe treatment option for treating PAE.

\section{Key words}

Acne vulgaris; Picosecond laser; Post-acne erythema 


\section{INTRODUCTION}

Acne vulgaris is a chronic skin disease of pilosebaceous units with multifactorial pathogenesis that affects about $80 \%$ of adolescents and young adults. ${ }^{1}$ Post-acne erythema (PAE), also referred to as post-inflammatory erythema (PIE), is one of the major problems during or after the treatment of acne vulgaris with any therapeutic modality. ${ }^{2}$ These lesions may resolve over time, but commonly persist for long times, sometimes lasting for several months. ${ }^{2}$ A variety of therapeutic options for PAE has been described with variable clinical outcomes and side effects. ${ }^{3-5}$ However so far, there is no standard treatment guidelines for PAE due to a limited numbers of published clinical studies. Recently, novel picosecond lasers have been developed for the treatment of variable indications. In the present report, we treated the patient with PAE using 595-nm picosecond-domain neodymium:yttriumaluminum-garnet (Nd:YAG) laser. This wavelength is generated with a hand piece containing a solid dye cylinder, pumped by the 532 -nm frequency-doubled Q-switched beam of the 1064-nm Nd:YAG laser. After four sessions of treatment, the patient presented nearly complete improvement of the lesions with no remarkable side effects or recurrence over the duration of 3 months.

\section{CASE REPORT}

A 21-year old, Korean woman presented to our clinic with multiple erythematous patches on the both cheek of her face (Fig. 1A). The patient was clinically diagnosed with PAE which persisted for several months after acne vulgaris and was scheduled to undergo laser treatment. After obtaining written informed consent, the patient was treated with total 4 sessions of bi-weekly exposure of 595$\mathrm{nm}$ picosecond-domain Nd:YAG laser (Picoplus; Lutronic Corp., Goyang, Korea). After cleansing patient's face, we performed laser treatment with sliding technique $15-\mathrm{mm}$ spot size, $0.1 \mathrm{~J} / \mathrm{cm}^{2}$ fluence with 450 picoseconds of exposure time) on both cheeks followed by additional pulse stacking technique $\left(5-\mathrm{mm}\right.$ spot size, $0.1 \mathrm{~J} / \mathrm{cm}^{2}$ fluence with 450 picoseconds of exposure time) on the red spotted area. The 595-nm laser was applied as low fluences and multiple passes to be a sub-purpuric fashion with the total number of approximately 1000 laser shots on the lesion. There was no systemic or topical anesthesia during the treatment and no prophylactic prescription such as systemic or topical corticosteroids for controlling possible post-laser inflammation or pigmentation. The patient was only recommended to apply moisturizers to support barrier recovery evoked by laser treatment. At 3 months after the last treatment, the patient presented nearly complete improvement of the PAE lesions without worsening or recurrence (Fig. 1B).

\section{DISCUSSION}

Acne vulgaris affects almost 80 percent of adolescents and young adults which can result in many adverse cutaneous consequences including post-acne erythema, post-
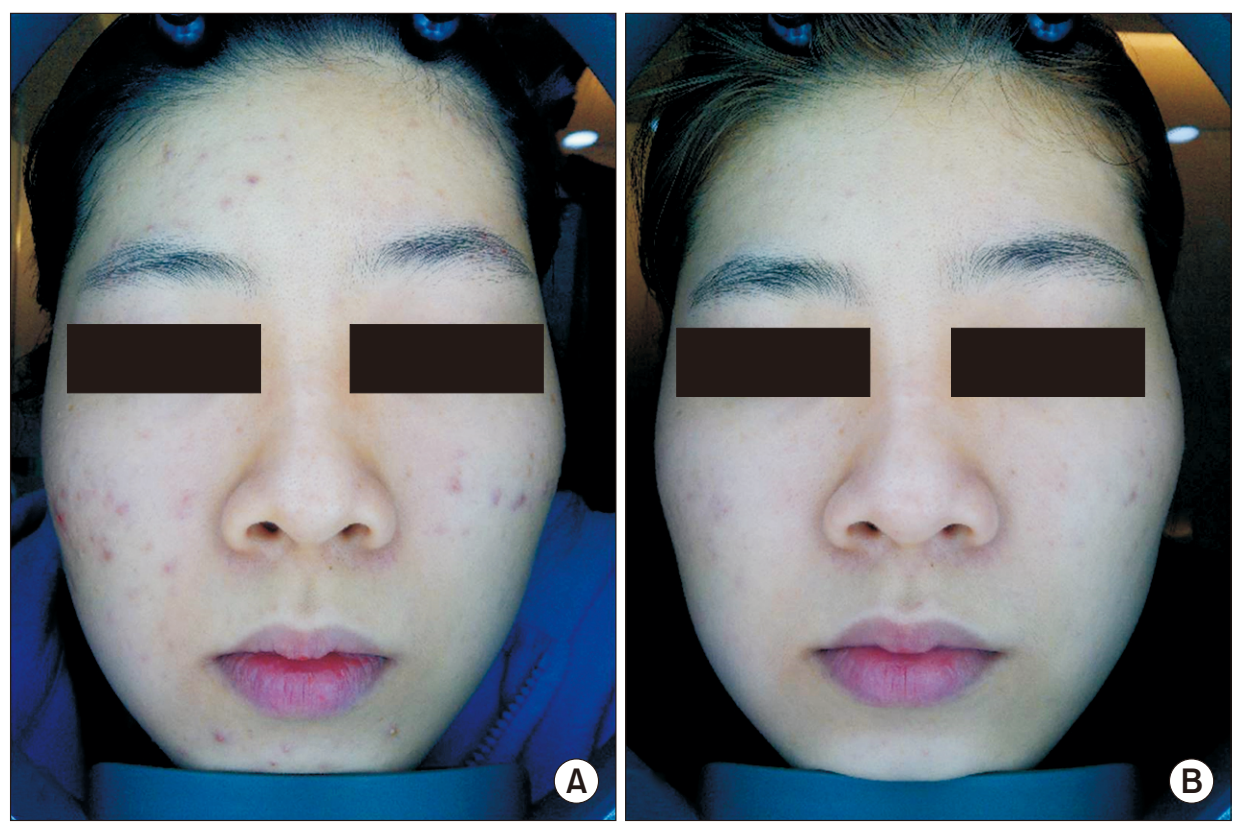

B
Fig. 1. Clinical improvement of the post-acne erythema after treatment with four sessions of $595-\mathrm{nm}$ picosecond-domain neodymium: yttrium-aluminum-garnet $(\mathrm{Nd}$ : YAG) laser: (A) before the treatment, (B) after four sessions of laser treatment. 
inflammatory hyperpigmentation (PIH), and scarring. 5 Therefore, the goals of acne therapy should not be limited only to achieve clearance of existing acne lesions, but also to prevent and manage possible sequelae. ${ }^{2}$

PAE is one of the most problematic cosmetic concerns in acne patients. ${ }^{2}$ These lesions usually resolve over time, but the long-lasting PAE is quite common, sometimes lasting for several months to years. ${ }^{7}$ However, the exact mechanism for this phenomenon after treatment of acne has not been elucidated. ${ }^{8}$

PAE is postulated as being due to wound healing-related dilatory changes in microvascular structures in the superficial dermis. Usually, these microvascular structures are visible as general redness. In addition, the epidermis is under the process of healing after tissue injury and is therefore thinner, allowing light to be reflected off easily the dilated microvasculature. Moreover, some believed that PAE is also caused by exogenous stimuli, such as the application of certain kinds of topical agents. ${ }^{2}$

A variety of therapeutic options for PAE has been described with variable clinical outcomes and side effects. Unfortunately, there is no standard treatment due to the limited number of published clinical studies. ${ }^{4,10}$ The 585$\mathrm{nm}$ wavelength is commonly used in vascular treatment with long pulse durations measured in milliseconds, and it targets the endothelium and wall structure of various vessels. At this wavelength, the absorption by melanin and oxyhemoglobin is very high. For this reason, pulsed dye laser (PDL) treatment is the most widely used method for the treatment of blood vessels. ${ }^{4.9}$ However, purpura would be a potential side effect associated with PDL treatment of any vascular related lesion and the induction of purpura can increase the possibility of PIH formation. Additionally, cryogen cooled devices can be another risk factor for PIH in darker skin types. ${ }^{10}$

Considering the postulated size of the microvascular structures in PAE, shorter pulse widths could be applied to vascular treatment. Previously, nanosecond-domain Q-switched Nd:YAG laser wavelength of $585 \mathrm{~nm}$ has been reportedly used for vascular treatment associated with PAE. It can be inferred that repeated irradiation shorter than the thermal relaxation time may actually be effective in treating micro-vasculature. However, this wavelength has a limited effect due to its relatively shallow penetration. In this case, we used picosecond-domain Q-switched laser at the 595- $\mathrm{nm}$ wavelength. This wavelength is actually slightly better absorbed in oxyhemoglobin than $532 \mathrm{~nm}$ but less well-absorbed in melanin. Under this circumstance, target chromophore can be treated effectively without interference from competing chromo- phore. This wavelength can be treated up to the lesions in the relatively deep area, and due to the nature of picosecond-domain laser, even low fluence can generate rather enough energy to achieve the optimal therapeutic outcomes with relatively safe profile. We were unable to observe any clinical signs of direct vascular destruction in this case, such as purpura, since the erythema appearing after acne treatments usually does not appear as vascular structures visible to the naked eye.

Recently, novel picosecond lasers have been developed for the treatment of variable indications. Picosecond-domain lasers were deemed to generate faster photothermal reactions, more extensive photomechanical effects, and less injury to adjacent structures than nanoseconddomain lasers. However, there is little report about the treatment of vascular lesions by using picoseconddomain laser. To the best of the authors' knowledge, this is the first case of the effective treatment of PAE using a 595-nm picosecond-domain Nd:YAG laser. The 595-nm laser in the present study was applied as low fluences and multiple passes in a sub-purpuric fashion. There was no systemic or local side effect during or after the treatment and no further prophylactic prescription to prevent possible post-laser downtime was needed excepting the use of moisturizers. This made the patient more comfortable than another types of treatment usually followed by post-treatment medications.

In conclusion, low-fluence 595-nm picosecond-domain Q-switched Nd:YAG laser treatment is safe and effective for the treatment of PAE. The advantages of this new laser treatment compared to others are less pain, greater patient comfort and almost no downtime. Therefore the low-fluence 595-nm picosecond-domain Q-switched $\mathrm{Nd}$ :YAG laser could be considered as an adjuvant or alternative treatment options in patients with persistent PAE. However, our current one is a descriptive case report which did not reveal the appropriate treatment interval, enough energy, and the mechanism of treatment. Further studies on the underlying mechanisms and clinical effects of PAE treatment using picosecond domain laser will be needed.

\section{CONFLICT OF INTEREST}

The authors report no conflicts of interest.

\section{REFERENCES}

1. Lee HJ, Kim YJ, Lee SJ, Lee CH, Choi EH, Lee SH, et al. Acne vulgaris successfully treated with long-pulsed 1,064-nm 
neodymium:yttrium-aluminum-garnet laser. Med Lasers 2018;7:91-3.

2. Panchaprateep R, Munavalli G. Low-fluence $585 \mathrm{~nm}$ Qswitched Nd:YAG laser: a novel laser treatment for post-acne erythema. Lasers Surg Med 2015;47:148-55

3. Glaich AS, Goldberg LH, Friedman RH, Friedman PM. Fractional photothermolysis for the treatment of postinflammatory erythema resulting from acne vulgaris. Dermatol Surg 2007;33:842-6.

4. Yoon HJ, Lee DH, Kim SO, Park KC, Youn SW. Acne erythema improvement by long-pulsed 595-nm pulsed-dye laser treatment: a pilot study. J Dermatolog Treat 2008;19:38-44.

5. Wang B, Wu Y, Luo YJ, Xu XG, Xu TH, Chen JZ, et al. Combination of intense pulsed light and fractional $\mathrm{CO}(2)$ laser treatments for patients with acne with inflammatory and scarring lesions. Clin Exp Dermatol 2013;38:344-51.

6. Rocha MA, Costa CS, Bagatin E. Acne vulgaris: an inflammatory disease even before the onset of clinical lesions. Inflamm Allergy Drug Targets 2014;13:162-7.
7. Bae-Harboe YS, Graber EM. Easy as PIE (Postinflammatory Erythema). J Clin Aesthet Dermatol 2013;6:46-7.

8. Harper JC. An update on the pathogenesis and management of acne vulgaris. J Am Acad Dermatol 2004;51:S36-8.

9. Park KY, Ko EJ, Seo SJ, Hong CK. Comparison of fractional, nonablative, 1550-nm laser and 595-nm pulsed dye laser for the treatment of facial erythema resulting from acne: a splitface, evaluator-blinded, randomized pilot study. J Cosmet Laser Ther 2014;16:120-3.

10. Munavalli GS, Weiss RA, Halder RM. Photoaging and nonablative photorejuvenation in ethnic skin. Dermatol Surg 2005; 31:1250-60.

How to cite this article: Kim JH, Choe SJ, Kim TG. Post-acne erythema successfully treated with 595-nm picoseconddomain neodymium:yttrium-aluminum-garnet laser. Med Lasers 2020;9:84-87. https://doi.org/10.25289/ML.2020.9.1.84 